\title{
EL ALGORITMO DE LA HECHICERÍA. ANÁLISIS CUANTITATIVO Y CUALITATIVO DEL ARCHIVO INQUISITORIAL TOLEDANO*
}

\author{
RoBerto Morales EstéveZ \\ ESERP Madrid \\ prof.rmorales@eserp.com
}

\section{INTRODUCCIÓN}

$\square$ n diciembre de 1971, el Consejo de Investigaciones Científicas danés amplió la ayuda concedida al historiador y especialista en cultura folklórica de la Universidad de Copenhague Gustav Henningsen y a su investigador ayudante Jaime Contreras. En 1972 ambos pusieron en marcha un proyecto para recoger la actividad de los tribunales inquisitoriales a partir de las relaciones de causa enviadas a la Suprema por los inquisidores (Jimeno Aranguren, 2012: 31).

La investigación se materializó en un artículo de Henningsen (1977), que fue complementado por otro a cargo de Jean-Pierre Dedieu (1978), dedicado en exclusiva al archivo inquisitorial toledano. Estos datos han sido utilizados de manera sistemática por multitud de estudios posteriores con carácter cuantitativo, aunque muestran sus limitaciones en tanto en cuanto no evidencia los casos reales sobre los que el historiador puede trabajar, esto es, los procesos que descansan realmente en nuestros archivos. Fajardo Spínola (1999: 17) asevera que las relaciones de causa abarcan el periodo 1540-1700, a pesar de que hay algunas posteriores, y que en las series existen muchas lagunas. Estos inconvenientes no desacreditan ni restan valor a todos los trabajos que se han basado en los análisis

\footnotetext{
* Este artículo se ha desarrollado dentro del proyecto «La mujer frente a la Inquisición española y novohispana» (FEM2016-78192-P), I+D de Excelencia del Ministerio de Economía y Competitividad (MINECO), financiado por la Agencia Estatal de Investigación (AEI) y el Fondo Europeo de Desarrollo Regional (FEDER, UE); y del grupo de investigación «Mentalidades mágicas y discursos antisupersticiosos (siglos XVI, XVII y XVIII)», grupo consolidado por la Universidad Autónoma de Madrid.
} 
de Dedieu y Henningsen, toda vez que ambos historiadores no han dejado de mejorar y reajustar sus conclusiones.

No obstante, para mis investigaciones centradas en la documentación existente en el Archivo Histórico Nacional (AHN), me vi obligado a realizar una actualización de las mismas. Con la creación de una base de datos he pretendido dotar a la comunidad científica y a cualquier persona interesada en el tema de un listado fiable sobre los casos juzgados en el archivo toledano. Hemos comenzado abordando delitos como la bigamia y principalmente la hechicería - materia en la que se centra este trabajo.

La base del mismo parecía obvia en tanto en cuanto es la que a día de hoy ofrece el AHN en formato físico. Se trata del Catálogo de causas contra la fe seguidas ante el Tribunal del Santo Oficio de la inquisición de Toledo, publicado en 1903 y que actualmente sigue vigente.

La confección del catálogo relata la historia y las vicisitudes del propio registro inquisitorial. El archivo toledano descansaba originariamente en el Archivo Central de Alcalá de Henares, donde el jesuita Francisco Fresca comenzó con su rigurosa catalogación. Por Real Orden de 10 de febrero de 1897, los fondos pasaron a su actual ubicación en el Archivo Histórico Nacional, lugar en el que Miguel López Campillo procedió a la preparación de la catalogación del padre Fresca para su publicación con prólogo del director del archivo, el valenciano Vicente Vignau (1903, VI).

La base de datos confeccionada para la realización del presente estudio sigue el modelo del padre Fresca con algunas modificaciones surgidas tras la comparación de las referencias aportadas por el jesuita que ofrece en el Portal de Archivos Españoles (PARES) <http://pares.culturaydeporte.gob.es/inicio.html>. Una de las ampliaciones ha sido reseñar el cambio de signatura que se ha producido y que ha ocasionado más de un disgusto al investigador al consultar el fondo toledano. También hemos complementado la información que de los reos ofrece el padre Fresca con los ofrecidos en PARES, para contar con una base de datos lo más completa posible, aunque somos conscientes de que nada sustituye la consulta individualizada de todos los legajos que custodia el Archivo Histórico Nacional.

Igualmente, hemos aprovechado para sacar lo que podríamos llamar «casos ocultos», es decir, procesos que se guardaban bajo una sola signatura y nombre. Con ellos, hemos tenido especial cuidado en no dar por supuesto la pena impuesta que recibió el encausado principal de la misma, dejando la casilla de «Resultado de la causa» como «no consta». Ejemplo de ello es el sumario de Alonso de Montenegro Rojas (AHN, Inq. 91, exp. 11). En dicha causa se encierran los expedientes de su hermana Marina, Francisca de Virueña, Juan de Frías, carpintero, Bernardino de Cuellar y Teresa, mujer de Alonso Sedeño y las declaraciones contra todos ellos de la beata Catalina González. 


\begin{tabular}{|c|c|c|c|c|c|c|c|c|c|c|c|c|c|c|c|c|c|c|c|c|c|c|c|c|c|c|c|c|c|c|c|c|}
\hline & 울 & a & - & 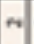 & 이 & 이 & $\rightarrow$ & - & 0 & 이. & - & 이 & - & 이 & - & ㅇ․ & $\rightarrow-$ & $\rightarrow$ & & 이 & 이 & 이 & 이 & - & & o. & 아 & $\circ$ & - & 0 & 0. & o \\
\hline 4 & 히 & n & $=$ & $\pi$ & $\backsim$ & 8 & $\pi$ & $\sim$ & o. & $\Rightarrow$ & $\bullet$ & 의 & a & 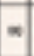 & $\bullet$ & & $r=$ & 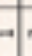 & & m. & 이 & $\pi$ & $\infty$ & & $\nabla$ & $\pi$ & 이. & - & - & & & \\
\hline & 8 & & $m$ & a & $\omega$ & e) & $\vec{T}$ & $\infty$ & $\cdots$ & $\Rightarrow$ : & $=$ & ta & ค & $\mathbb{R}$ & 오 & e. & $\Rightarrow r$ & & & 7 & $\infty$ & o & o & 0 . & 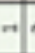 & $\infty$ & & 4 & $\Rightarrow$ & o. & $A$ & 0 \\
\hline & ₹ & & $m$ & $\mu$ & $\rightarrow$ & $\Rightarrow$ & 0 & 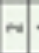 & $\rightarrow$ & . & - & $\sim$ & 12 & N & ค & & $\leadsto$ & 4 & & - & 2. & 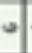 & $\rightarrow$ & H. & a & a & & +1 & 2 . & $n$ & & - \\
\hline $\mathrm{g}$ & 울 & se & - & 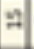 & $\infty$ & 0 & $N$ & $\mathrm{~m}$. & - & -1 & $=$ & $\approx$ & $\Rightarrow$ & $z$ & 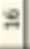 & &. \pm & $\infty$, & -4 & m & $\infty$ & - & 0 & o. & $\rightarrow$ & 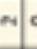 & - & N. & $\rightarrow$ & 0. & -10 & a \\
\hline c & a & & - & $n$ & $\varphi$ & $A$ & $\mathrm{~A}$ & - & $\mathrm{E}$ & 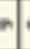 & $\cdot$ & \pm & $\approx$ & 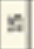 & R & $\sim$ & $t=$ & $\mathrm{m}$. & $\Rightarrow$ & 이: & 9 & 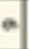 & $\varphi$ & $\rightarrow$ & $\mathrm{a}$ &. \pm & •| & $\backsim$ : & a. & n. & & 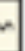 \\
\hline & ipusding: & a & $\circ$ & $\Rightarrow$ & - & 0 & 0 & $\circ$ & 0 & 0 & 0 & $=$ & 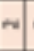 & $n$ & + & & $A$ & , & $m$ & + & $\pi$ & o & $\rightarrow-1$ & $\omega$ & n. & $m \cdot$ & 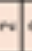 & 임. & $\bullet$ & $*$. & $\rightarrow$, & $m$ \\
\hline$y$ & *p+ranes & - & 이 & o & 0 & o & 0 & o. & o. & 이. & - & o. & $\circ$ & 이 & - & ㅇ. & $\rightarrow c$ & ㅇ. & o. & 이 & 이 & 인 & 0 & $\circ$ & o. & 0. & 이 & 이, & - & o. & 0. & 이 \\
\hline 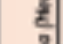 & cwues on & 0 & - & + & -4 & $n$ & 0 & o. & - & $m$ & - & $\rightarrow$ & $m$ & $\infty$ & - & o. & $\Rightarrow c$ & ㅇ. & ㅇ․ & $r$ & - & - & - & & o & o. & 아. & -1 & $\rightarrow$ & o. & o. & et \\
\hline & maje. & o & $m$ & $\rightarrow$ & - & $\rightarrow$ & $\vec{t}$ & $\omega$ & o. & ㅇ. & 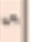 & $\rightarrow$ & \$) & a & ๑ & o. & - & $\mathrm{m}$, & & + & + & - & -1 & $\infty$ & - & 4 & - & $\backsim$, & $\infty$ & 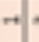 & & + \\
\hline & epenepues & $\rightarrow$ & - & $\Rightarrow$ & $\infty$ & $=$ & - & $\sim$. & - & 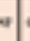 & a. & a) & A & $=$ & $A$ & -1 & $=-$ & 4 & o. & + & $\bullet$ & $\pi$ & $\approx$ & o. & - & A. & o. & ㅇ. & a. & o. & 0 & 이 \\
\hline & mianien & o & - & 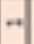 & $\Rightarrow$ & o & 0 & o. & - & 0 & o & o. & -4 & 이 & o & - & o. 5 & 0. & o. & 이 & 이 & 이 & 0 & - & o. & $\infty$. & - & 이 & $m$ & 0 & & o \\
\hline & lang: & e & - & 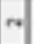 & 0 & - & $\Rightarrow$ & o. & - & o. & - & - & $\Rightarrow$ & - & $n$ & $m$ & $n$ & $n$, & $\sim$ & $m$ & 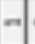 & - & $\Rightarrow$ & m. & n &.- & - & - & $\bullet$ & - & 4. & m \\
\hline & yetanpes: & $\circ$ & - & - & 이. & o & 0 & o. & o. & 이. & o & o. & 0 & 이 & - & - & $\rightarrow c$ & ㅇ. & o. & 이 & 이 & 이 & o) & o. & o. & 0. & o. & 이. & 0 . & o. & ㅇ. & c \\
\hline & ous en & 0 & 이 & -4 & - & $n$ & 0 & o. & o. & $\infty$ & o & - & $m$ & - & - & ㅇ․ & $-c$ & o. & ㅇ․ & $n$ & - & 이 & 0 & $\Rightarrow$ & o. & 0. & 아. & - & - & o. & o. & - \\
\hline 8 & mapduearue & o & - & . & 0 & $\sim$ & $\sim$ & $\sim$. & o. & . & $=$ & 9 & ร & $\approx$ & $n$ & o. & $n$ & $\mathrm{~m}$. & - & e & ना & - & $\infty$ & & & $\sim$. & -1 & $\bullet$ & $\infty$ & $\Rightarrow$ & -1. & - \\
\hline & openenpos & $m$ & 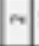 & 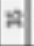 & 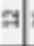 & 2 & A & $n$ & $\mathrm{~F}$ & $\infty ;$ & 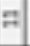 & a & $\approx$ & $x$ & ? & $n$ & $=2$ & $m$, & 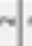 & $\sim$ & $\bullet$ & $n$ & $m$ & $\circ$ & $n$ & $\backsim c$ & 0 & 0 & $\approx$. & $\rightarrow$ & $\circ$ & 0 \\
\hline & स्पष & o & 이 & -4 & -1 & - & - & o. & $\sim$ & 이 & o & ㅇ․ & - & 이 & - & 아. & 0 & o. & o. & 아 & - & o & 0 & 0 . & o. & 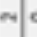 & 이, & o. & $m$ & 0. & 0. & o) \\
\hline & 포 & o & - & o & - & o & o & o. & - & 。. & - & o. & - & 이 & 이 & - & of & o. & o. & of & 이 & 이 & - & e. & o. & -. & o. & - & - & o. & o. & 이 \\
\hline & - & - & - & $\approx$ & 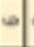 & 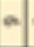 & 0 & $\sim$. & - & . & $\mathrm{m}$ & $\mathrm{z}$ & $\nabla$ & $\approx$ & 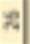 & $\sim:$ & $\mathrm{A}$ & •. & $\sim$ & $\infty$ & $\infty$ & 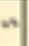 & $\infty$ & $=$ & $\bullet$ & $a$. & 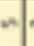 & $m$ & $\approx$ & - & & m \\
\hline & $\mathbf{z}$ & 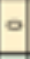 & - & $\omega$ & $\infty$ & $n$ & - & $\sim$. & 0. & - & $n$ & - & $n$ & 우 & 요 & - & $m$ & $m$ & o. & $\mathrm{Na}$ & था & - & 4 & $\Rightarrow$ & 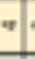 & $\leadsto$. & - & $\mathrm{m}$ & m. & 0. & - & 이 \\
\hline 8 & $\pi$ & 0 & 이 & 0 & 0 & - & 0 & o. & $\Rightarrow$ & 0 & 0 & o & 0 & - & - & 이 & 0. & 0. & o. & 이, & 0 & 이 & - & 0 & 0 & 0. & 0 & 0 & - & 0 & 0. & 0 \\
\hline & - & & - & $\pi$ & $\bullet$ & $x$ & - & $\sim$. & - & e. & $=$ & $\mathrm{n}$ & a & $\Rightarrow$ & A & -1 & $\pi$. & $\sim$ & $\sim$ & a & $\infty$ & • & $\infty$ & - & a & a. & . & $m$ & $A$ & - & $A$. & - \\
\hline & $\mathbf{z}$ & & - & $\approx$ & $\infty$ & \& & $\mathbb{A}$ & n. & $\circ$ & 4 & $\approx$ & 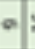 & \pm & $=$ & $\approx$ & $\rightarrow$ & $\Rightarrow$ & 4 & $m$. & 4 & 4 & n & 4 & $\omega$ & $n$ & $\bullet$ & - & 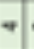 & $\mathrm{m}$ & $N$ & $\boldsymbol{N}$ & \\
\hline & ne1 & $m$ & $=$ & 8 & $\approx$ & 7 & A & $\sim$ & $x$ & 9 & R & $\pi$ & 8 & $\$$ & *8) & $\omega$ & $z=$ & a & $\infty$ & a & a) & - & $\bullet$ & $=$ & 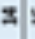 & \pm . & •. & $\sim$ & 8 & $n$ & - & \\
\hline & no & o & 이 & o & $\circ$ & o & o & 0 & 0 & 이. & o & o & 0 & 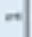 & - & 0 & $\circ 6$ & 0 & o. & o & - & o & - & 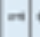 & o & $\circ$ & o & $\circ$ & - & o & $\circ$ & 이 \\
\hline & $\ln _{3} s$ & & - & B & $n$ & ค & $x$ & -1 & a. & 4 & • & a & \pm & 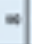 & $\bullet$ & $\rightarrow$ & $=$ & -4. & $\rightarrow$. & 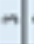 & - & - & $H$ & -4 & + & +4 & ㅇ․ & $\rightarrow$, & - & $\omega$ & $\rightarrow$. & \\
\hline & ne & & 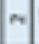 & 2 & - & 3 & $\rightarrow$ & -1 & $\cdots$ & •. & 3 & \pm & 8 & D. & R & $M$ & -7 & $\infty$. & $\sim$ & 요 & a & 0 & 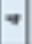 & $m$ & 요 & $\exists$ A. & $=$ & $\bullet$ & 8 & $m$ & • & \\
\hline & & ? & క్ & ₹्र & ह्र & ऐ) & ¿ & ; & $\sum$ & , & క్ & క్ర & 5 & 5 & है & 알 & $x>$ & 5 & 한. & ह्रा & ह्र & ट्र & हद्र & द्र. & द्र & द्वर & द्र. & c) ? & ह्र & ${ }_{c}$ & $\frac{8}{2}$ & \\
\hline & 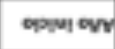 & & 8 & 8 & 3 & 옥 & 3 & R & 8 & 8 & छ & 5 & 8 & है] & $\mathrm{g}$ & 8 & 就 & ?요 & $\$$ & 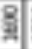 & 8 & है & $g$ & $\S$ & $\mathrm{AB}$ & $2:$ & \$) & $\pi$ & 8 & 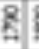 & 8 & \\
\hline
\end{tabular}

[GRÁfico 1]. Cuadro-resumen de la base de datos de los delitos de bigamia y hechicería en el Archivo inquisitorial de Toledo. Elaboración propia.

Edad de Oro, XXXIX (2020), pp. 43-56, ISSN: 0212-0429 - ISSNe: 2605-3314 
Del mismo modo, hemos incluido la información con referencia a qué legajos se encuentran disponibles en red y cuáles no para mayor utilidad de la base de datos. En estos momentos, se tienen catalogados 541 sujetos pertenecientes a los delitos de bigamia y hechicería, sorprendiendo la enorme diferencia de casos en red entre uno y otro delito. Mientras que los procesos de la bigamia casi el 100\% de ellos están disponibles para consultar en red, los de hechicería apenas llegan al 50\%.

Tras el vaciado de fuentes, contamos con un total de 353 casos de hechicería que se extienden entre 1524 - con el expediente de José Navarro (AHN, Inq. 92, exp. 14) - y 1818-1819, con el caso de Vicenta García (AHN, Inq. 83, exp. 3). Cuando comparamos dicha tabla con el estudio ya clásico de Jean-Pierre Dedieu (1978) basado en las «Relaciones de causas», elevan la cifra a 373 casos de hechicería, un 5,17\% del total de los 7.216 casos que llevó a cabo el tribunal de Toledo, mientras que nuestros 353 casos registrados, arrojan un porcentaje de un 4,89\%, con respecto a las cifras de Dedieu.

\section{Delimitando el término de heChicería: a Vueltas Con UnA REalidad ESQuiva}

La hechicería siempre se ha ligado a la brujería e incluso en las fuentes contemporáneas una y otra actividad mágica han sido confundidas. Este equívoco se refleja en obras como la de Antonio de Torquemada y su Jardín de Flores curiosas (1575: 303), «... aunque ay mugeres que son hechiceras y no bruxas, como se podrá bien ver en Lucio Apuleyo, De asino aureo, las que son bruxas son todas hechiceras». Martín del Río, cuando trata sobre ello en su obra Disquisitionum magicarum libri $V I^{1}$, deja claro que ambas son practicantes de magia, pero distingue a brujas de hechiceras en el momento en el que relata el sabbat de las brujas.

Como fenómeno atemporal y universal, la hechicería mantiene una constante tanto en los rituales como en el instrumental, a pesar de la diferencia cronológica y geográfica, algo de lo que ya se apercibió Frazer, en su celebérrimo libro $L a$ rama dorada, en el que catalogó los distintos tipos de magia, simpática o por contacto. Frente a la hechicería como fenómeno intemporal, la brujería como concepto se fue conformando a lo largo de la Edad Media en Occidente y cristalizó de manera casi definitiva en el Malleus maleficarum a finales del siglo xv.

Ambas actividades son consideradas herejía, ya que en cualquier caso lo que se realiza es un pacto diabólico. Explícito en la brujería, o implícito en los procesos de las hechiceras. Este pacto diabólico es uno de los elementos principales de lo que Levack (1995: 53-98) llamó «concepto acumulativo de brujería». Mientras que la bruja nace, la hechicera se hace en un contexto cultural de magia popular

1 Para una panorámica de esta obra y de otros manuales contra las brujas véase Zamora Calvo (2018: 163-180). 
aceptada por muchos. En palabras de Henningsen: «La brujería no es un arte o técnica mágica que se pueda aprender, sino un poder sobrenatural innato u adquirido mediante trato con un ser sobrenatural» (2014: 133).

La brujería — como ya definió el maestro Caro Baroja (1966: 148) — es un fenómeno comunal y del ámbito rural, a diferencia de la hechicería que es individual y de carácter urbano, de carácter manual y práctico. Este carácter comunal comentado por Caro Baroja es lo que, a juicio de Campagne (1997: XXVI), es el elemento central que justifica y explicita la caza de brujas.

En España, la brujería en su vertiente satánica — salvo contadas excepcionesse circunscribe, según Joseph Pérez (2010: 72), a las provincias vascas, Navarra, Pirineo aragonés y catalán, mientras que la hechicería se encuentra en toda la península española. García Cárcel parece no compartir el modelo geográfico de Pérez aduciendo que las situaciones de la brujería y la hechicería son difícilmente adscribibles a pautas geográficas o antropológicas determinadas (2000: 292-293). Aun así, plantea un modelo de categorización; en la montaña impera la bruja y en el llano lo hace la hechicera. En esta categorización, la bruja de nuevo demuestra tener un carácter más tribal, con escasa romanización y de cristianización tardía.

La bruja está especializada en asesinatos de niños ${ }^{2}$, sequías y catástrofes. Entretanto, la hechicera está más próxima a la stregha italiana. Esta se caracteriza por pertenecer a una sociedad abierta y de mayor movilidad. La hechicera es más individualista y funcionalista y se vuelca en la práctica de magia amorosa o la curación de enfermedades.

Esta última es la más habitual en España y concretamente la que se encuentra con mayor asiduidad en el archivo toledano. Como asevera Pérez: «... hubo muchas hechiceras para solucionar problemas personales, pero pocas brujas a quienes achacar los males colectivos de la nación» (2002: 206).

\section{UN DELITO FEMENINO}

En lo que todos los autores coinciden es en el carácter femenino, tanto si hablamos del delito de hechicería como si lo hacemos de brujería ${ }^{3}$. Así lo corrobora nuestro análisis realizado de los legajos inquisitoriales toledanos en el Archivo Histórico Nacional, como se puede apreciar en la siguiente tabla, y que nos sitúan en la media europea según los datos aportados por Levack (1995: 178):

2 Según Campagne, frente a la bruja europea, que nunca hizo del infanticidio su actividad principal, la española se asocia casi de manera excluyente con el asesinato de niños (2009: 157-ss.).

3 Para una panorámica sobre las mujeres y su tratamiento jurídico por parte de la Inquisición, véase Collantes de Terán de la Hera (2017: 105-145). 


\begin{tabular}{|l|c|c|}
\hline $\begin{array}{c}\text { Total de causas de hechicería } \\
\text { por sexo 1524-1819 }\end{array}$ & Número & Porcentaje \\
\hline Mujeres & 263 & $74,50 \%$ \\
\hline Hombres & 89 & $25,21 \%$ \\
\hline Hermafroditas & 1 & $0,28 \%$ \\
\hline
\end{tabular}

[TABLA 1]. Total de causas juzgadas por el tribunal inquisitorial toledano entre 1524 y 1819 en relación a su sexo. Fuente: AHN. Elaboración propia.

Martín de Castañega perfila aún más a las servidoras del demonio, ya fuesen brujas o hechiceras:

E mas son de las mujeres viejas e pobres que de las moças e ricas porque como despues de viejas, los hombres no hacen caso dellas, tienen recurso al demonio se cumplen sus apetitos, en especial si quando moças fueron inclinadas e dadas al vicio de la carne; a estas semajantes engaña el demonio quando viejas, prometiéndoles de cumplir sus apetitos e cumpliendolos por obra, como adelante se dira. E mas ay de las pobres y necessitadas porque, como en otros vicios, la pobreza es muchas vezes ocasión de muchos males en personas que no la toman en voluntad o en paciencia; por esto, pensando que el demonio suplira sus necesidades o responderá a sus desseos y apetitos, mas son engañadas las viejas e pobres que no las moças e las que tienen bien lo que ha menester, porque les da a entender que no les faltaba nada si a el siguen (1994 [1529]: 21).

Nuestra base de datos corrobora tal apreciación. Abundan en la misma las mujeres viudas, sin oficio claro. Las más numerosas de ellas relacionadas con trabajos de escasa cualificación casi siempre de la industria textil (costurera de ropa blanca, hiladora de oro y plata, lavandera, corredora de prendas, calcetera, etc., o relacionadas con el mundo de la prostitución). De las Cuevas incide en tal apreciación al aseverar que entre estas mujeres destacan algunas características como son la carencia de recursos económicos, con un nivel cultural mínimo y desprotegidas (1980: 55).

Ninguno de los casos recogidos recuerda en ningún momento a la poderosa mujer soñada por Michelet en su ya mítica obra La bruja de 1862. Impregnada de romanticismo y feminismo a partes iguales (Campagne, 2009: 63), la obra fue redactada en apenas seis meses, sin documentación e investigación previa. La bruja puede leerse hoy más como evocadora novela que como ensayo histórico (Cohn, 1987: 146). Por ello sorprende que a pesar de que todos los trabajos académicos incidan en esa pobreza e indefensión de «las brujas y hechiceras», aún hoy a nivel popular y en trabajos de escasa calidad científica, siga triunfando la 
imagen de la bruja como mujer empoderada y transgresora de la cultura heteropatriarcal. La sombra de Michelet es alargada.

Las hechiceras del archivo toledano no residen en los bosques donde celebran improbables sabbats. Abundan en el mentidero madrileño de San Felipe, bulliciosas gradas donde encontraremos gente del hampa, germanías y, en general, la aristocracia del crimen (Lisón Tolosana, 1990: 23). Es allí, en este mundo de lumpen, pobreza y crimen donde encontraremos por regla general a las hechiceras que intentan escapar de su estado de extrema necesidad por medio de la estafa más o menos consciente ofreciendo sus servicios.

Por lo general, la hechicería es un fenómeno urbano y Madrid es buena prueba de ello a pesar de que solo de los 84 vecinos encausados por hechicería dos sean naturales de la ciudad. La emigración del campo a la ciudad es algo habitual en la España Moderna (Tausiet, 2007) y Madrid no es una excepción, al canalizarse en ella toda la corriente migratoria del interior, sobre todo de las dos Castillas (De las Cuevas Torresano, 1980: 60). Alvar Ezquerra ofrece un dato en extremo calificador del fenómeno migratorio para la villa y corte al afirmar que la población estable de Madrid entre 1560 y 1600 se multiplica por nueve (1989: 50). Se trata de una población con una mentalidad rural que se está urbanizando (Tausiet, 2007: 200-201).

El escenario cambia cuando analizamos a los hombres. Estos suelen aparecer más relacionados con la magia culta y libresca, totalmente distinta de la cultura mágica de las mujeres vinculada a la tradición oral y a la cultura popular.

De entre los 89 encausados varones destacan dos colectivos principalmente: el de afines con el mundo de la salud, caso del boticario Juan Bautista de Salazar (AHN, Inq. 95, exp. 11), de Francisco Romano (AHN, Inq. 94, exp. 17), barbero de la familia del infante cardenal o del herbolario Juan de la Comba (AHN, Inq. 94, exp. 10). De otra parte estaría el estamento eclesiástico, entre los que podemos citar al clérigo Juan Fernández (AHN, Inq. 86, exp. 24), o al clérigo presbítero Jaime Manobel (AHN, Inq. 90, exp. 6) involucrado de la misma manera, como muchos de sus compañeros, en artes astrológicas. Fuera de esos colectivos, la muestra de ocupaciones entre los hombres es muy heterogénea. Cabría destacar los relacionados con la industria textil y agrícola, lo que no es óbice que entre ellos se incluya de manera excepcional el catedrático de matemáticas de la universidad de Salamanca, el licenciado González (AHN, Inq. 87, exp. 12).

A este predominio de la hechicería femenina solo podemos contraponer la excepción del único capítulo de brujería que, siguiendo a De las Cuevas Torresano (1980), se da en el momento de mayor auge de la persecución del tribunal de Toledo, en la primera mitad del siglo XviI. Ocurrió en la villa de Malagón (Ciudad Real) en el año 1625, en el que tras leerse en el mes de abril 
un edicto de fe, acabaron encarceladas cinco mujeres ${ }^{4}$ acusadas de hechicería. Un capítulo de brujería más propio de los Pirineos, cuyos legajos son poco claros, confusos según De las Cuevas Torresano (1980: 78), y acaban con sentencias que van poco más allá de ser reprendidas y advertidas que no vuelvan a sus actividades brujeriles.

Hasta la llegada del afamado caso de Zugarramurdi, España estuvo exenta en líneas generales de capítulos de cazas de brujas homologables al resto de Europa por las instrucciones inquisitoriales del 14 de diciembre de 1526 (Henningsen, 2014: 143).

Precisamente lo ocurrido en Zugarramurdi cerró cualquier posibilidad a que la Inquisición se viera envuelta en una caza de brujas. El abogado de las brujas, Salazar y Frías, envió un informe a la Suprema que desactivó lo que podría haber sido una de las histerias brujeriles más funestas bajo la jurisdicción inquisitorial española. El órgano supremo inquisitorial recogió todas las sugerencias de Salazar y Frías y firmó el 29 de agosto de 1614 las nuevas instrucciones que llegarían al tribunal de Logroño el 18 de septiembre (Henningsen, 2010: 431). El principio del fin de la persecución en España había comenzado y marcaría la actuación del Santo Oficio sobre tan delicado tema hasta la desaparición del mismo.

\section{ANÁlisis de LAS SENTENCIAS}

Para analizar las penas debemos de partir del hecho de que no todos los que se libran de la condena por hechicería salían sin más del tribunal. Cabe la posibilidad de que el acusado lo fuera de varios delitos, entre ellos, hechicería de la que podía salir absuelto, pero no así de otras acusaciones. Así le ocurrió a Elena/o de Céspedes ${ }^{5}$, que libre de la acusación de hechicería, tuvo que hacer frente a la pena impuesta por embustes al hacerse pasar por hermafrodita, vestir como hombre, además de burlarse del santo sacramento del matrimonio. Por ello, tuvo que salir en auto de fe y recibir 200 azotes además de ser recluida en un hospital diez años.

4 Son las siguientes: Catalina Parrila (AHN, Inq. 93, exp. 3), la morisca Inés Naranjo (AHN, Inq. 93, exp. 13), María Marta (AHN, Inq. 90, exp. 12), Ana Hernández Naranja (AHN, Inq. 88, exp. 2) y María Brava (AHN, Inq. 83, exp. 4).

5 AHN, Inq. 234, exp. 24. Sobre este fascinante caso de transexualidad en la España Moderna existe los estudios clásicos de Barbazza (1984: 17-40). Para un estudio más profundo, la referencia indiscutible sigue siendo la obra de Maganto Pavón (2007). Sobre el discurso médico de esta encausada contamos con el sobresaliente trabajo de Moral de Calatrava (2018: 230-243). 


\begin{tabular}{|l|c|c|}
\hline \multicolumn{1}{|c|}{ Sentencias 1524-1819 } & Número & Porcentaje \\
\hline Absueltos & 9 & $2,55 \%$ \\
\hline Condenados & 157 & $44,47 \%$ \\
\hline Incompletas & 102 & $28,89 \%$ \\
\hline No consta & 28 & $7,93 \%$ \\
\hline Sobreseídas & 1 & $0,28 \%$ \\
\hline Suspendidas & 56 & $15,86 \%$ \\
\hline TOTAL & 353 & $100,00 \%$ \\
\hline
\end{tabular}

[TABLA 2]. Total de sentencias del tribunal inquisitorial de Toledo. Fuente: AHN. Elaboración propia.

Todos los autores coinciden en la benignidad de las penas impuestas a los encausados por hechicería frente a las durísimas penas que se le imponía a judíos conversos protestantes (García Cárcel, 1980: 243). Henningsen llega a decir que tal benignidad debió resultar un escándalo para la mentalidad justiciera del norte de Europa (2014: 145). Prueba de esta benignidad es que en el siglo XVII, momento álgido de la actividad inquisitorial contra la hechicería, ninguno de los acusados fue relegado a la hoguera (De las Cuevas Torresano, 1980: 53) .

Otra buena prueba de ello es la actitud de la Inquisición catalana y sus esfuerzos por contener la persecución en el siglo XVII de la justicia ordinaria, que en muchas ocasiones derivaba en una verdadera caza de brujas. En palabras de Castell Granados: «Las últimas investigaciones sobre el caso catalán confirman el papel destacado de la justicia ordinaria en dicha persecución, en claro contraste con la actuación de unos representantes del Santo Oficio atrapados entre el escepticismo y la impotencia» (2017: 52) ${ }^{7}$.

En la misma línea, Blázquez Miguel aseveraba años antes que en Cataluña solo entre 1600 y 1630 fueron quemadas no menos de cuatrocientas mujeres, y que no fueron más por la pronta intervención del Santo Oficio (1990: 98).

$6 \quad$ Esto contrasta con la información que aporta Levack de otras zonas de Europa, donde el número de ejecutadas se mueve entre el 16\% de Finlandia y el $90 \%$ de Pays de Vaud. Tanto en Italia como en España, las ejecuciones se realizaron principalmente en el siglo XVI (1995: 48-49).

7 El mismo autor abre su trabajo con una cita de lo más elocuente sobre el alcance de la caza de brujas llevada a cabo en Cataluña y los infructuosos intentos de la Inquisición por detenerla: «En este Principado de Cataluña de dos o tres años a esta parte an ahorcado los jueces seglares más de treçientas personas por brujas [...] Háçenles sus procesos y aunque dellos resulta el delito y crimen de la heregía y apostasía, no lo remiten a este Santo Officio» (2017: 51). Carta del fiscal de la Inquisición en Barcelona a la Suprema, 1619 (Biblioteca Nacional de España, ms. 2.440, f. 140r). 
Las penas impuestas a los profesionales de la magia, hombres o mujeres ${ }^{8}$, casi nunca llegaron a ser de galeras o cárcel perpetua. Casi siempre encontramos penas menores como destierros, multas, abjuraciones, ayunos u oraciones (Cirac Estopañán, 1942: 230). Esta benignidad, para suerte de los encausados, podría deberse a que la Inquisición, tras la hechicería y la magia, viera la realidad de la misma. Todo engaño y estafa por parte de gente necesitada de recursos sin otra opción que la de delinquir en una España sumida en una crisis económica. Como asevera María Tausiet (2007), la hechicería se consideró una suerte de oficio prohibido comparable a la alcahuetería - las «celestinas castellanas» de Cirac Estopañán (1942: 39) - , la prostitución o la venta ilegal.

Esto me quedó patente tras analizar uno de los casos más llamativos del archivo toledano, como lo fue el del clérigo nigromante Jaime Manobel (AHN, Inq. 90, exp. 6), que mediante una teatralización de un pacto demoniaco propio del Fausto de Marlowe, se esconde un embaucador, un pícaro que pretendió sobrevivir mediante engaños y, por tal cosa, llegó a ser condenado por la Inquisición.

Por tanto, ¿cabría hablar de racionalismo de la Inquisición española? Pérez no termina de verlo claro:

Si la Inquisición española se mostró indulgente con las brujas, a diferencia de los tribunales laicos del resto de Europa, no fue por su condición racionalista o positivista, menos aún compasiva ante las desgracias del pueblo llano, sino sencillamente porque siempre se atuvo a la doctrina tradicional del catolicismo, tal como había sido definida en el Canon Episcopi, en el siglo x (2010: 279).

Cuando analizamos el número de encausados por hechicería por décadas, destaca de manera notable el siglo XVII y sobre todo las décadas entre 1620 y 1640 , entre las cuales se juzgaron a 128 personas. En solo tres decenios se juzgaron 45 personas más que en el siglo precedente. De las 353 que fueron juzgadas en tres siglos, nada menos que un $36,2 \%$ lo fueron en las décadas mencionadas. Es interesante hacer notar que este aumento de sentencias no fue correlativo al número de condenados, ya que en el siglo XvI lo fueron un $68,87 \%$ de los reos, frente a un $55,46 \%$ de entre 1620 y 1640 .

8 Hago esta apreciación porque no era lo habitual. Como dice Collantes de Terán: «Mientras que en el derecho civil la fragilitas e imbecilitas sexus se tradujo en restricciones a la capacidad de obrar de la mujer, en el derecho penal le supuso un tratamiento indulgente que aproximaba su conducta a la de los niños y a la de los débiles mentales. Los propios juristas determinaron que el sexo era una justa causa para moderar la pena ordinaria a las mujeres mediante la imposición de una pena arbitraria inferior a la establecida, pudiendo llegar incluso a la exoneración de cualquier castigo». Incluso «en ocasiones llega a justificar en ella comportamientos que nunca toleraría a los hombres» (2017: 108-110). 


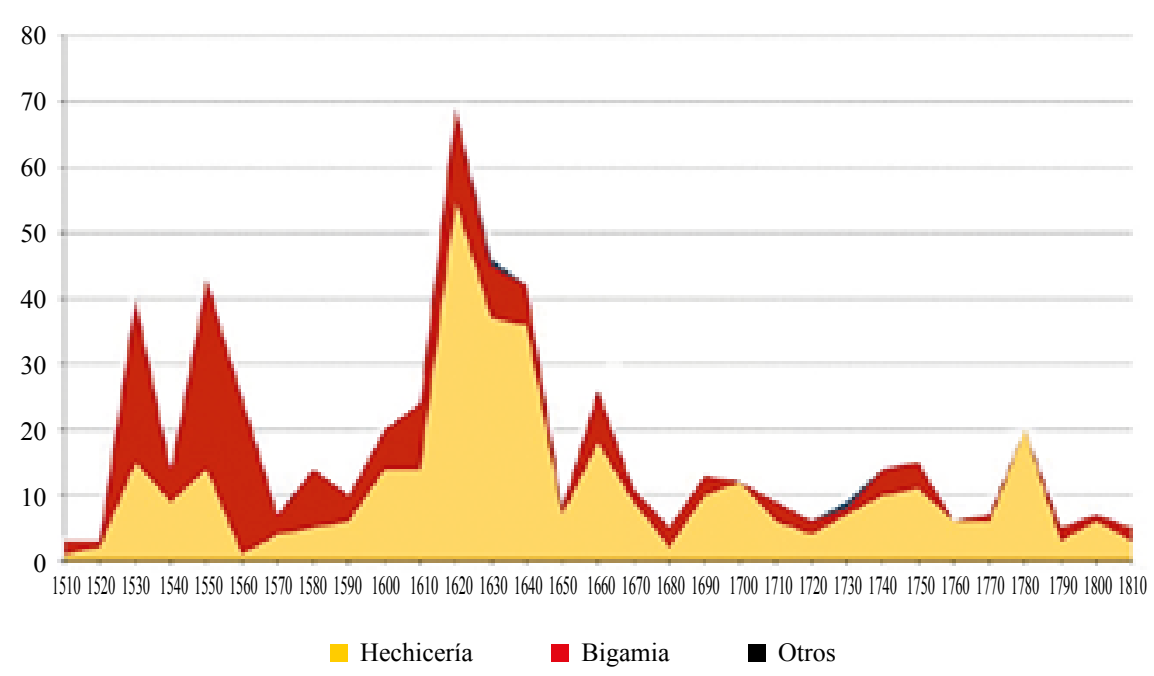

[GRÁfico 2]. Encausados por delito de bigamia y hechicería por décadas. Fuente: AHN. Elaboración propia.

Este notable aumento no obedece a razones locales, sino que se incardina en la dinámica europea de caza de brujas que, según Delemeau, alcanzó «su paroxismo la locura perseguidora entre 1560 y 1630» (2012: 434).

\section{ConClusión}

Los datos que nos muestra el archivo inquisitorial toledano expresan que el delito de hechicería es eminentemente urbano y femenino, donde la presencia de la brujería de corte satánico es inexistente. Los números de causas y el sexo de los encausados son homologables a muchas zonas de Europa, no así las sentencias, que como se ha reflejado son más laxas, ya que la Inquisición española entendió la brujería a través de presupuestos tradicionales católicos.

La principal valía de este trabajo reside en la elaboración de una base de datos anteriormente expuesta, más completa y actualizada que en anteriores ocasiones, aunque más limitada geográficamente. Entendemos que la puesta a disposición de tal banco de datos a la comunidad científica hará del vaciado de fuentes del fondo inquisitorial una labor más sencilla. 
BiBLIOGRAFÍA

Alvar EzQuerra, Alfredo (1989). El nacimiento de una capital europea. Madrid entre 1561 y 1606. Madrid: Turner.

Barbazza, Marie-Catherine (1984). «Un caso de subversión social. El proceso de Eleno de Céspedes (1587-1589)». Criticón, 26, pp. 17-40.

Blázquez Miguel, Juan (1990). Madrid. Judios, herejes y brujas. El Tribunal de Corte (1650-1820). Madrid: Arcano.

Campagne, Fabián A. (2009). Strix hispánica. Demonología cristiana y cultura folklórica en la España moderna. Buenos Aires: Prometeo libros.

Caro Baroja, Julio (1966). Las brujas y su mundo. Madrid: Alianza Editorial.

Carvajal GonzÁlez, Helena (2019). "'Cient sacras de pargamino’: Un impreso 'sine notis' desconocido del taller zaragozano de Jorge Coci en el Archivo Histórico Nacional». Revista general de información y documentación, 29, 2, pp. 413-425.

CASTAÑEga, fray Martín de (1994). Tratado de las supersticiones y hechicerías y de la possibilidad y remedio dellas [1529]. Juan Robert Muro Abad (ed.). Logroño: Gobierno de la Rioja / Instituto de Estudios Riojanos.

CAstaÑEga, fray Martín de (1997). Tratado de las supersticiones y hechicerías. Fabián Alejandro Campagne (ed). Buenos Aires: Universidad de Buenos Aires.

Castell Granados, Pau (2017). «“Con toda templança y moderación”. El Santo Oficio ante la caza de brujas en Cataluña». En María Jesús Zamora Calvo (ed.), Mulieres inquisitionis. La mujer frente a la Inquisición en España. Vigo: Editorial Academia del Hispanismo.

Cirac Estopañán, Sebastián (1942). Los procesos de hechicería en la Inquisición de Castilla la Nueva (Tribunales de Toledo y Cuenca): Aportación a la Historia de la Inquisición española. Madrid: Instituto Jerónimo Zurita / Centro Superior de Investigaciones Científicas.

CoHn, Norman (1987). Los demonios familiares de Europa. Óscar Cortés Conde (trad.). Madrid: Alianza Editorial.

Collantes de TerÁn DE la Hera, María José (2017) «Particularidades del proceso inquisitorial por razón de sexo». En María Jesús Zamora Calvo (ed.), Mulieres inquisitionis. La mujer frente a la Inquisición en España. Vigo: Editorial Academia del Hispanismo, pp. 105-145.

Dedieu, Jean-Pierre (1978). «Les causes de la foi de l'Inquisition de Tolède (1483-1820)». Mélanges de la Casa Velázquez, 14, pp. 143-171.

De las Cuevas Torrresano, M. a de Luz (1980). «Los procesos inquisitoriales de hechicería en el tribunal toledano durante la primera mitad del siglo XVII». Anales toledanos, 13, pp. 25-92.

Fajardo SpínOla, Francisco (1999): «La actividad procesal del Santo Oficio. Algunas consideraciones sobre su estudio». Manuscrits, 17, pp. 97-117.

Frazer, James George (2003). La rama dorada. Elizabeth y Tadeo I. Campuzano (trads.). Madrid: Fondo de Cultura Económica.

García CÁrCel, Ricardo (1980). Herejía y sociedad en el siglo XVI. La Inquisición en Valencia (1530-1609). Barcelona: Ediciones Península.

García CÁrcel, Ricardo y Doris Moreno (2000). La Inquisición: Historia crítica. Barcelona: Temas de Hoy. 
Henningsen, Gustav (1977). «El banco de datos del Santo Oficio. Las relaciones de causa de la Inquisición Española (1500-1700)». Boletín de la Real Academia de la Historia, CLXXIV, pp. 547-570.

HeNningsen, Gustav (2010). El abogado de las brujas. Brujería vasca e Inquisición española. Madrid: Alianza Editorial.

HenNingsen, Gustav (2014): «La inquisición y las brujas». eHumanista. Journal of Iberian Studies, 26, pp. 133-152.

Jimeno ARANGUREN, Roldán (2012) «Gustav Henningsen o el porqué del estudio de la Inquisición». Revista de Estudios Vascos, 9, pp. 22-39.

LeVACK, Brian P. (1995). La caza de brujas en la Europa Moderna. José Luis Gil Aristu (trad.). Madrid: Alianza Editorial.

Lisón Tolosana, Carmelo (1990). Demonios y exorcismos en los Siglos de Oro. La España mental I. Madrid: Akal Universitaria.

Maganto Pavón, Emilio (2007). El proceso inquisitorial contra Elena/o de Céspedes (15871588). Biografia de una cirujana transexual del siglo XVI. Madrid: Método Gráfico.

Martínez Millán, José (2009). La Inquisición española. Madrid: Alianza Editorial.

Moral de Calatrava, Paloma (2018) «"En buena mediçina”. Las fuentes médicas del discurso de Céspedes ante la Inquisición». Asclepio: Revista de Historia de la Medicina $y$ de la Ciencia, 70, 2. pp. 230-243. DOI: https://doi.org/10.3989/asclepio.2018.14.

Michelet, Jules (2004). La bruja. Un estudio de las supersticiones en la Edad Media. Rosina Lajo y M. ${ }^{\text {a }}$ Victoria Frígola (trads.). Madrid: Akal.

Morales Estévez, Roberto (2014). «Los grimorios y recetarios mágicos: del mítico Salomón al clérigo nigromante». En Eva Lara y Alberto Montaner (coords.), Señales, portentos y demonios. La magia en la literatura y la cultura españolas del Renacimiento. Salamanca: Semyr, pp. 535-554.

PÉREZ, Joseph (2002). Crónica de la Inquisición española. Barcelona: Martínez Roca.

PÉrez, Joseph (2010). Historia de la brujería en España. Madrid: Espasa.

TAusiet, María (2007). Abracadabra Omnipotens. Magia urbana en Zaragoza en la Edad Moderna. Madrid: Siglo XXI.

TORQUEMADA, Antonio de (1575). Jardin de flores curiosas, en que se tratan algvnas materias de Humanidad. Philosophia, Theologia y Geographia, con otras cosas curiosas y apacibles. Compuesto por Antonio de Torquemada. Dirigido al Muy Ilustre y Reverendisimo Señor Don Diego Sarmiento de Sotomayor, Obispo de Astorga. Va hecho en seis tratados, como aparece en la tercera página de esta obra. Anveres: Casa de Juan Corderio.

Zamora Calvo, María Jesús (2018). «Tratado contra las brujas en la Biblioteca Nacional de España». Documenta \& Instrumenta, 16, pp. 163-180. DOI: http://dx.doi. org/10.5209/rev DOCU.2018.v16.60234.

VV. AA. (1903). Catálogo de las causas contra la fe seguidas ante el tribunal de Santo oficio de la inquisición de Toledo, y de las informaciones genealógicas de los pretendientes á oficios del mismo: con un apéndice, en que se detallan los fondos existentes en este archivo de los demás tribunales de España, Italia y América. Madrid: Tipografía de la Revista de Archivos, Bibliotecas y Museos / Archivo Histórico Nacional.

Recibido: 31/03/2020

Aceptado: 15/05/2020

Edad de Oro, XXXIX (2020), pp. 43-56, ISSN: 0212-0429 - ISSNe: 2605-3314 


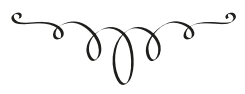

EL ALGORITMO DE LA HECHICERÍA.

ANÁLISIS CUANTITATIVO Y CUALITATIVO DEL ARCHIVO INQUISITORIAL TOLEDANO

RESUMEN: El presente estudio nace de la creación y análisis de una base de datos sobre el delito de hechicería del tribunal inquisitorial de Toledo a lo largo de toda su historia. Tras delimitar el siempre esquivo término de hechicería, los datos han sido analizados desde el punto de vista de género y el resultado de las sentencias ofrecidas por el propio portal de Archivos Españoles (PARES) y el catálogo de causas contra la fe seguidas ante el tribunal del Santo Oficio de la Inquisición de Toledo de principios del siglo XIX. Con ello se ha pretendido actualizar los trabajos y resultados, principalmente de Henningsen y Dedieu, con los que contábamos, que al basarse en las relaciones de causa son algo menos fiables. Palabras ClaVe: Estadística, Inquisición, Toledo, hechicería, brujería.

The Sorcery Algorithm.

Quantitative and Qualitative Analysis of Toledo Inquisitorial Archive

ABSTRACT: The following study comes from the creation and analysis of a database on witchcraft crime from the Toledo Inquisitorial Court through the ages. Once defined the always difficult term witchcraft, data has been analysed from a gender point of view and the result of the sentences obtained from the Spanish Files web (PARES) and the index of lawsuits against Faith performed by the Toledo Inquisition's Holy Ofice Court at the beginning of the 19th century. All this is intended to perform an update on the existing works and data, mainly from Henningsen and Dedieu, that by being based on cause relations, are less reliable as a result.

KeY WORDS: Stadistic, Inquisition, Toledo, Witchcraft, Sorcery. 